\title{
Matéria
}

ISSN 1517-7076

Revista Matéria, v. 11, n. 4, pp. 385 - 394, 2006

http://www.materia.coppe.ufrj.br/sarra/artigos/artigo10794

\section{Propriedades Mecânicas e Termomecânicas de Compósitos com Partículas de Diamante Dispersas em Matriz Epoxídica Modificada na Razão Resina/Endurecedor}

\author{
Sergio N. Monteiro, Gustavo W. de Menezes, Ana Lúcia D. Skury, Felipe P. D. Lopes, Rubén J. S. \\ Rodriguez, Guerold S. Bobrovnitchii
}

Universidade Estadual do Norte Fluminense, UENF, Laboratório de Materiais Avançados, LAMAV, Av.

Alberto Lamego, 2000, 28013-602, Campos dos Goytacazes, Brasil.

e-mail: sergio.neves@ig.com.br, gwagner@uenf.br, lucia@uenf.br, felipeperisse@pop.com.br, sanchez@uenf.br, guerold@uenf.br.

\section{RESUMO}

Foram estudadas as propriedades mecânicas dos compósitos com partículas dispersas de diamante em matriz de resina epóxi curada com diferentes proporções de endurecedor caracterizadas pela razão resina/endurecedor. Investigou-se também o comportamento termomecânico através de ensaios de DMA (Análise Dinâmico-Mecânica). No presente trabalho foi feita uma completa avaliação das propriedades mecânicas e termomecânicas no amplo intervalo de razão resina/endurecedor associado a possíveis aplicações tecnológicas. Foram fabricados compósitos com até 30\% em peso de partículas de diamante dispersas em matriz epóxi do tipo DGEBA/TETA com diferentes razões resina/endurecedor, variando de 7 a 21. Para todas as condições investigadas a resistência diminuiu com a quantidade incorporada de diamante. Para matrizes com razão resina/endurecedor acima do estequiométrico 13, os compósitos apresentaram melhor desempenho mecânico. Os resultados dos ensaios termomecânicos mostraram que o módulo de armazenamento dos compósitos aumenta com a quantidade incorporada de diamante. Através da tangente delta foi possível avaliar prováveis mecanismos que contribuem para o desempenho termomecânico destes compósitos.

Palavras chaves: Compósitos, partículas de diamante, matriz epóxi, razão resina/endurecedor, análise térmica-DMA.

\section{Mechanical and Thermo-Mechanical Properties of Diamond Particles Dispersed in PHR Modified Epoxy Matrix Composites}

\section{ABSTRACT}

The mechanical properties of composites with dispersed diamond particles in epoxy matrix cured with different proportions of hardener to monomer ratio, characterized by the resin/hardener ratio (phr) were studied. An investigation on the thermo-mechanical behavior of these composites was also carried out by dynamical mechanical analysis (DMA). In the present work a complete evaluation of the mechanical properties was carried in a wide interval of phr associated with possible technological applications. Composites with up to $30 \mathrm{wt}$. \% of diamond particles dispersed in type DGEBA/TETA epoxy matrix were fabricated with phr ratios varying from 7 to 21. For all investigated conditions, the composite strength decreased with the amount of incorporated diamond. Matrices with phr above the stoichiometric 13 were associated with composites with better mechanical performance. The DMA results showed that the storage modulus increases with the amount of diamond particles incorporated in the composite. The values obtained for the delta tangent, allowed an evaluation of possible mechanisms that contribute to the thermal mechanical performance of these composites.

Keywords: Composites, diamond particles, epoxy matrix, phr ratio, DMA-thermal analysis. 


\section{INTRODUÇÃO}

Compósitos diamantados com matriz de epóxi vêm sendo recentemente investigados como possíveis materiais abrasivos para polimento de pedras ornamentais como mármore e granito [1-4] .

A vantagem de se utilizar a matriz de epóxi deve-se à sua versatilidade como um polímero termorrígido. As resinas epóxi apresentam boa fluidez antes da cura permitindo fácil processamento na temperatura ambiente. Após a cura estas resinas não sofrem grande retração o que permite reproduzir com maior fidelidade a geometria do molde. Mais importantes ainda são as superiores propriedades físicas e químicas associadas com distintos agentes que abrem o anel epóxi bem como as diferentes condições de cura, tempo e temperatura, além do percentual relativo da razão resina/endurecedor (phr) [్-]]. Em particular, quando aminas alifáticas são utilizadas como agente endurecedor, modificações da relação estequiométrica com a resina acarretam sensíveis variações no comportamento mecânico do sistema epóxi [7-ㅁ] . Especificamente, para sistemas formados pelo monômero epoxídico do tipo éter diglicidílico do bisfenol A, DGEBA com o endurecedor do tipo trietileno tetramina, TETA, um excesso deste último em relação à percentagem estequiométrica, phr 13, produz polímeros com elevada tenacidade [9, 10]. Verificou-se que para 17 partes de endurecedor por cem partes de resina, phr 17, tem-se condições otimizadas para as propriedades térmicas e mecânicas [11]. Entretanto, uma investigação abrangente das propriedades mecânicas de compósitos diamantados com matriz DGEBA/TETA dentro de uma gama de phr's ainda não foi realizada.

Em recentes publicações [1 $\underline{3}]$ investigou-se as propriedades termomecânicas de compósitos diamantados com matriz de epóxi. Nestes trabalhos variou-se a quantidade de diamante incorporado também a resinas DGEBA/TETA modificadas. Através de ensaios térmicos associados à análise mecânico-dinâmica, DMA, (“dynamic mechanical analysis") constatou-se que os compósitos diamantados com phr 17 são consideravelmente mais rígidos do que aqueles com phr 13. Com a incorporação de $30 \%$ em peso em partículas de diamante, ocorre um significativo aumento no módulo de armazenamento dos compósitos sobretudo naqueles com matriz de phr 17.

Tendo em vista este resultado, o presente trabalho estendeu a investigação para outros valores de phr, tanto com maior excesso de endurecedor, phr $>13$, quanto maior quantidade relativa de resina, isto é, phr < 13. A análise dos resultados dos ensaios de DMA não se limitou ao módulo de armazenamento mas abordou também o módulo de perda e a tangente delta $(\tan \delta)$.

Assim, o objetivo do presente trabalho foi realizar uma completa avaliação das propriedades mecânicas e termomecânicas destes compósitos diamantados no mais amplo intervalo de phr de interesse para aplicações tecnológicas como material abrasivo.

\section{MATERIAIS E MÉTODOS}

Os materiais utilizados foram partículas de diamante e resinas epóxi do tipo DGEBA/TETA modificadas relativamente à quantidade de endurecedor. As partículas de diamante foram produzidas no Setor de Materiais Superduros do Laboratório de Materiais Avançados da Universidade Estadual do Norte Fluminense, LAMAV/UENF, através da transformação catalítica de pó de grafite com pó de liga NiMn a $1250^{\circ} \mathrm{C}$ e pressão de $4,7 \mathrm{GPa}$ em um dispositivo de alta pressão do tipo bigorna conforme descrito em outro trabalho [12].

As resinas epoxídicas utilizadas como matriz constituídas de misturas do monômero DGEBA com proporções percentuais do endurecedor TETA correspondente ao intervalo de phr 7 a phr 21 associado à propriedades de interesse prático [7-9]. Composições de cada uma destas resinas com diferentes quantidades de 10, 20 e $30 \%$ em peso de partículas de diamante foram vigorosamente misturadas e vazadas em moldes abertos de silicone.

A cura destes compósitos diamantados foi realizada na temperatura de aproximadamente $25^{\circ} \mathrm{C}$ por uma semana. Corpos de prova de tração foram confeccionados com dimensões e características apresentadas anteriormente [4]. Após um leve polimento para aparar as saliências e ajustar suas dimensões, os corpos-deprova foram ensaiados em máquina Instron, modelo 5582, com uma taxa de deformação de 2,8x10-4 $\mathrm{s}^{-1}$. Pelo menos 6 corpos-de-prova foram ensaiados para cada composição, isto é, valor de phr da matriz e fração em peso de diamante.

Corpos-de-prova também foram ensaiados dinamicamente em um equipamento da TA Instruments, modelo DMA-2980, operando com frequiência de $1 \mathrm{~Hz}$ e uma taxa de aquecimento de $2^{\circ} \mathrm{C} / \mathrm{mim}$. Curvas de variação do módulo de armazenamento, E', módulo de perda, E”, e tangente delta, $\tan \delta$, foram diretamente obtidas dos ensaios de DMA.

A superfície da fratura dos corpos-de-prova foi analisada por microscopia eletrônica de varredura, MEV, em um microscópio Jeol, modelo JSM-6460 LV. Esta análise foi conduzida em amostras cortadas dos corpos-de-prova, previamente metalizadas com ouro, e observada nas imagens de elétrons secundários entre 15 e $25 \mathrm{kV}$. 


\section{RESULTADOS E DISCUSSÃO}

A Figura 1 ilustra o aspecto de uma curva de tração, carga vs. deslocamento, obtida diretamente da máquina Instron, para um dos compósitos diamantados com phr 21.

phr $21-20 \%$ diam

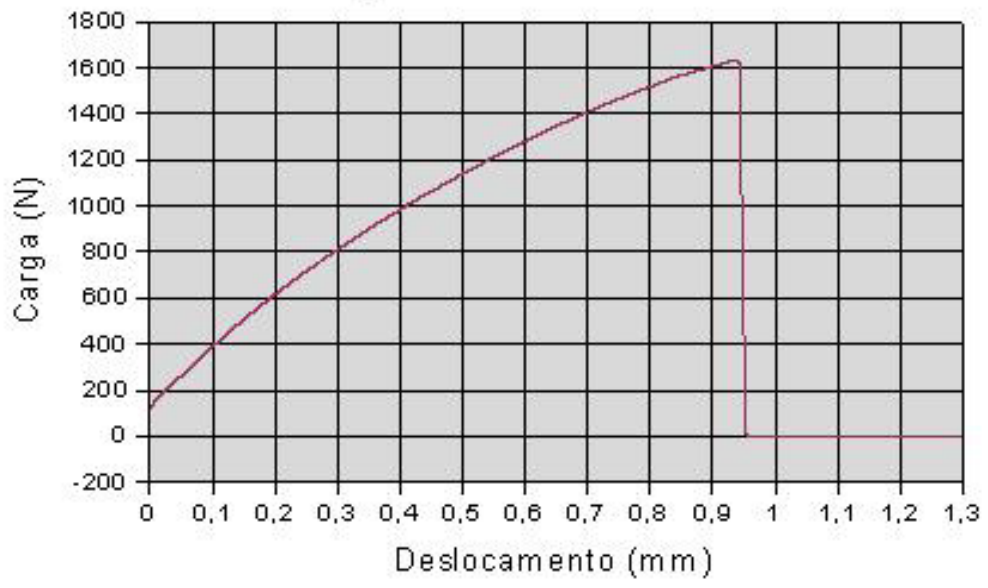

Figura 1: Curva típica de tração para um compósito diamantado com matriz epóxi DGEBA/TETA.

A Figura 2 apresenta a variação da resistência dos compósitos, medida pela tensão máxima em tração, com a fração em peso de partículas de diamante incorporadas à matriz epoxídica com diferentes valores de phr. Vale destacar nesta figura que, para todos os valores de phr, existe uma tendência de decréscimo na resistência à tração com a incorporação de diamante. No caso do phr 7, esta tendência é menos acentuada e, dentro dos limites de erro estatístico, poder-se-ia até considerar que praticamente não ocorreu variação.

Outro aspecto a ser destacado é o maior nível de resistência dos compósitos com phr 17 comparativamente aos outros, para qualquer fração incorporada de diamante. Isto corresponde a uma clara evidência de que uma relação endurecedor/resina pouco maior que a estequiométrica melhora o desempenho mecânico do compósito.

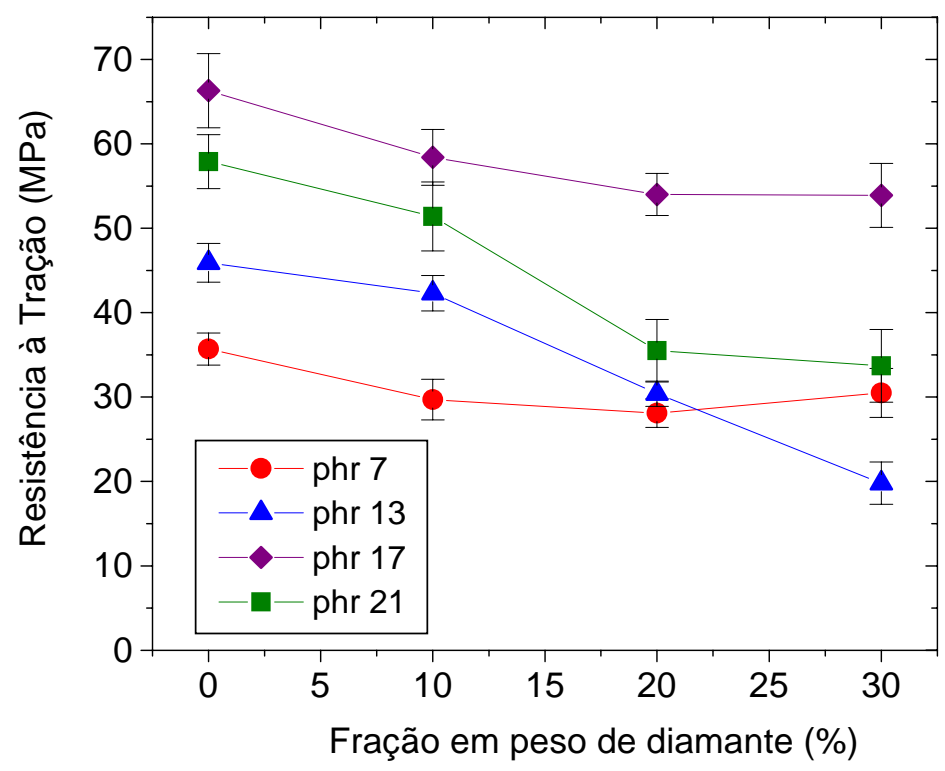

Figura 2: Variação da resistência à tração do compósito com a fração incorporada de diamante, para diferentes valores de phr. 
Em princípio, um decréscimo na resistência mecânica de um compósito devido à incorporação de partículas é decorrente de uma fraca ligação destas com a matriz. Neste caso, eventualmente poderá ocorrer descolamento da interface partícula/matriz para solicitações mecânicas correspondentes a tensões menores que a resistência da própria matriz. Nos pontos de descolamento tem-se uma falha que, em materiais relativamente frágeis, concentra tensões e pode rapidamente propagar trincas que levam à ruptura total do material.

Para os compósitos com matriz epoxídica DEGEBA/TETA modificada em relação ao phr, um excesso do endurecetor TETA, ou seja, phr $>13$, acarreta materiais com maior tenacidade [ $\underline{9}$, $\underline{10}]$. Isto explica os maiores níveis de resistência obtidos na Figura 2 para phr 17 e 21. Contrariamente, para phr 7 e 13, nesta mesma figura, têm-se relativamente menores valores de resistência. A maior fragilidade destas matrizes para phr $\leq 13$ associada à baixa resistência interfacial epóxi/diamante, facilita a nucleação e propagação de trincas em menores valores de tensão aplicada.

A Figura 3 apresenta os conjuntos correspondentes às relações phr 7, 13, 17 e 21 de curvas da variação do módulo de armazenamento, E', para compósitos com 0, 10, 20 e 30\% em peso diamante.Observa-se que, para todas as condições de phr, exceto phr 13, a maior fração, 30\%, incorporada de diamante resulta em maior valor de E' ao longo de todo intervalo de temperatura. Também é importante notar que os maiores níveis de E’ estão associados às curvas com phr 17 na Figura. 3(c).

Para avaliar quantitativamente a predominância dos compósitos com phr 17, em relação ao módulo de armazenamento, a Figura 4 apresenta a variação do valor de E', medido para a temperatura ambiente de $25^{\circ} \mathrm{C}$, em função do phr. Observa-se que o maior valor de E' = 5,7 GPa para 30\% de diamante corresponde a um pico em phr17 relativamente aos valores vizinhos. O motivo do maior valor de E' para phr 17 já foi discutido em outra publicação []].

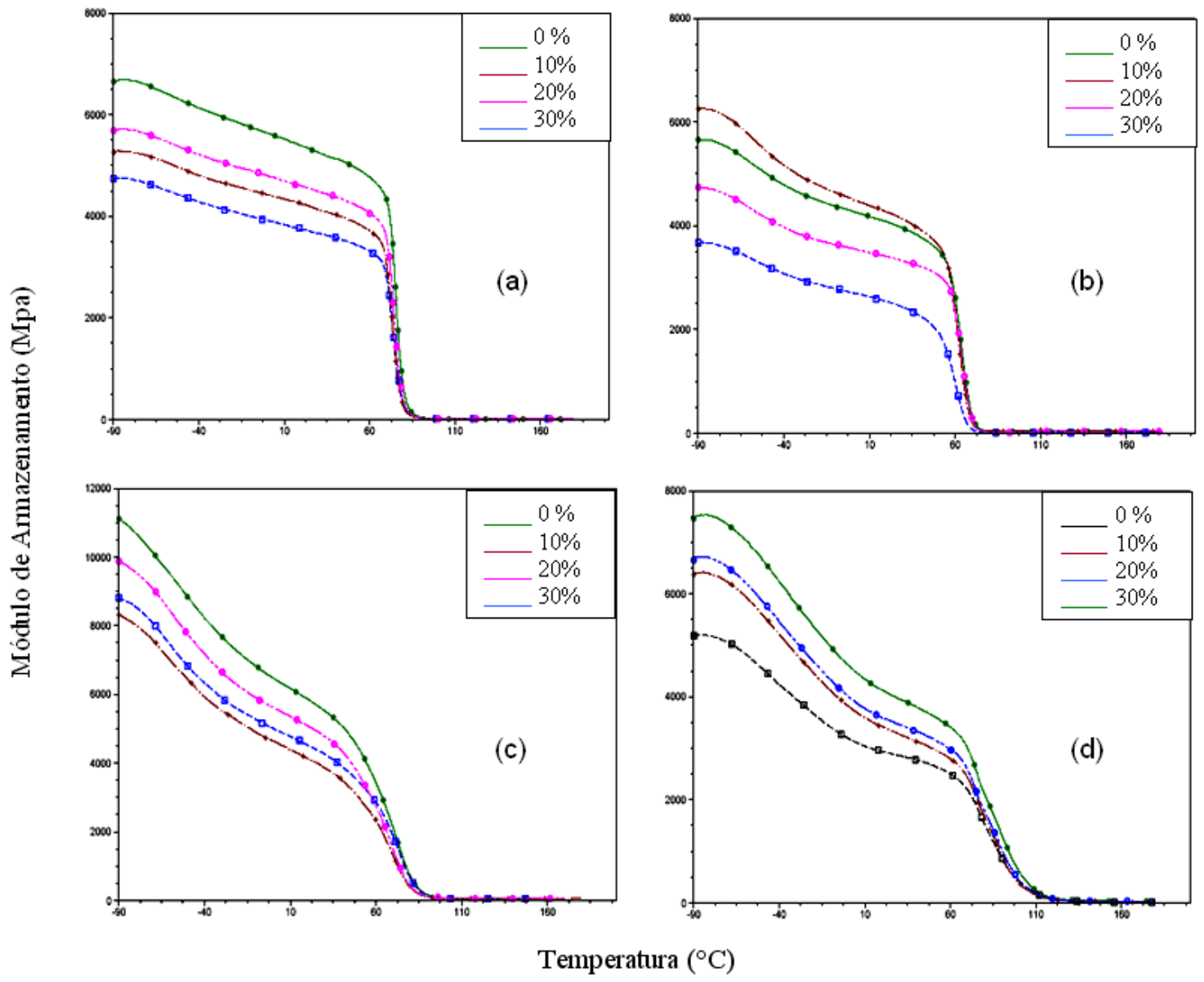

Figura 3: Curvas do módulo de armazenamento vs. temperatura para compósitos epoxídicos com diferentes frações em peso de partículas de diamante: (a) phr 7, (b) phr 13, (c) phr 17 e (d) phr 21. 


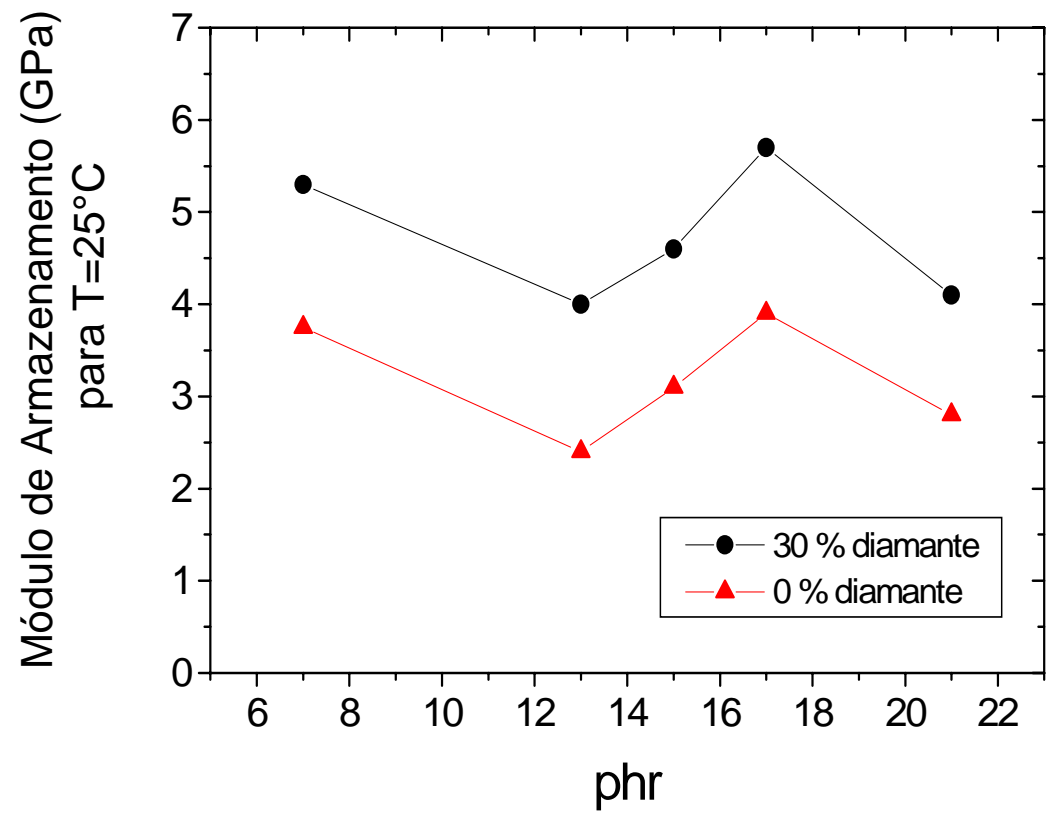

Figura 4: Variação do módulo de armazenamento, à temperatura ambiente de $25^{\circ} \mathrm{C}$, em função do phr.

Vale aqui lembrar que quando um material é submetido a uma solicitação mecânica oscilatória temse uma resposta dinâmica na qual a rigidez pode ser entendida como um módulo complexo [13, $\underline{14]}$ :

$$
\mathrm{E}^{*}=\mathrm{E}^{\prime} \cos \delta+\mathrm{i} \mathrm{E}^{\prime} \operatorname{sen} \delta
$$

onde E' armazena a energia fornecida como tensão aplicada e a devolve imediatamente quando se retira a tensão. Já E” corresponde à parcela de energia não devolvida quando se retira a tensão.

No caso do phr 17, Figura 4, a maior quantidade de endurecedor relativamente ao phr 13 estequiométrico está associada a um mecanismo que dificulta a mobilidade das cadeias moleculares e restringe a deformação plástica da matriz. Em conseqüência, a energia de uma solicitação mecânica poderá ser dissipada antes que ocorra uma propagação catastrófica de trincas [10] e o compósito torna-se relativamente mais tenaz.

A Figura 5 apresenta os conjuntos correspondentes às relações phr 7, 13, 17 e 21 de curvas de variação do módulo de perda, E”, para compósitos com 0, 10, 20 e 30\% em peso de diamante. Em particular, na Figura 5(d) estão superpostas as curvas de E', E” e tan $\delta$ para dar uma idéia conjunta de como se relacionam todas as curvas em relação à temperatura.

Como normalmente acontece em materiais poliméricos [14], as curvas do módulo de perda passam por máximos a baixas temperaturas, entre -90 e $-40^{\circ} \mathrm{C}$ nos presentes resultados, com tendência a maiores valores de E” para maiores quantidades de diamantes incorporadas. Seguem-se picos praticamente coincidentes, entre 60 e $90^{\circ} \mathrm{C}$, para todas as frações de diamantes em cada conjunto com mesmo phr. A temperatura de ocorrência destes últimos picos varia em um estreito intervalo relativamente ao phr, sendo menor, $63^{\circ} \mathrm{C}$, para o phr 13 e maior, $86^{\circ} \mathrm{C}$, para phr 21 .

Uma vez que o módulo de perda, Equação (1), está associado à energia visco-elástica retida no material [14], pode-se inferir que os compósitos investigados apresentam, abaixo de $60^{\circ} \mathrm{C}$, maior capacidade de armazenar energia quanto maior for a quantidade de diamante. Acima desta temperatura, ocorre a transição vítrea da matriz com efeito máximo caracterizado pelos picos entre 63 e $86^{\circ} \mathrm{C}$ na Figura 5. 

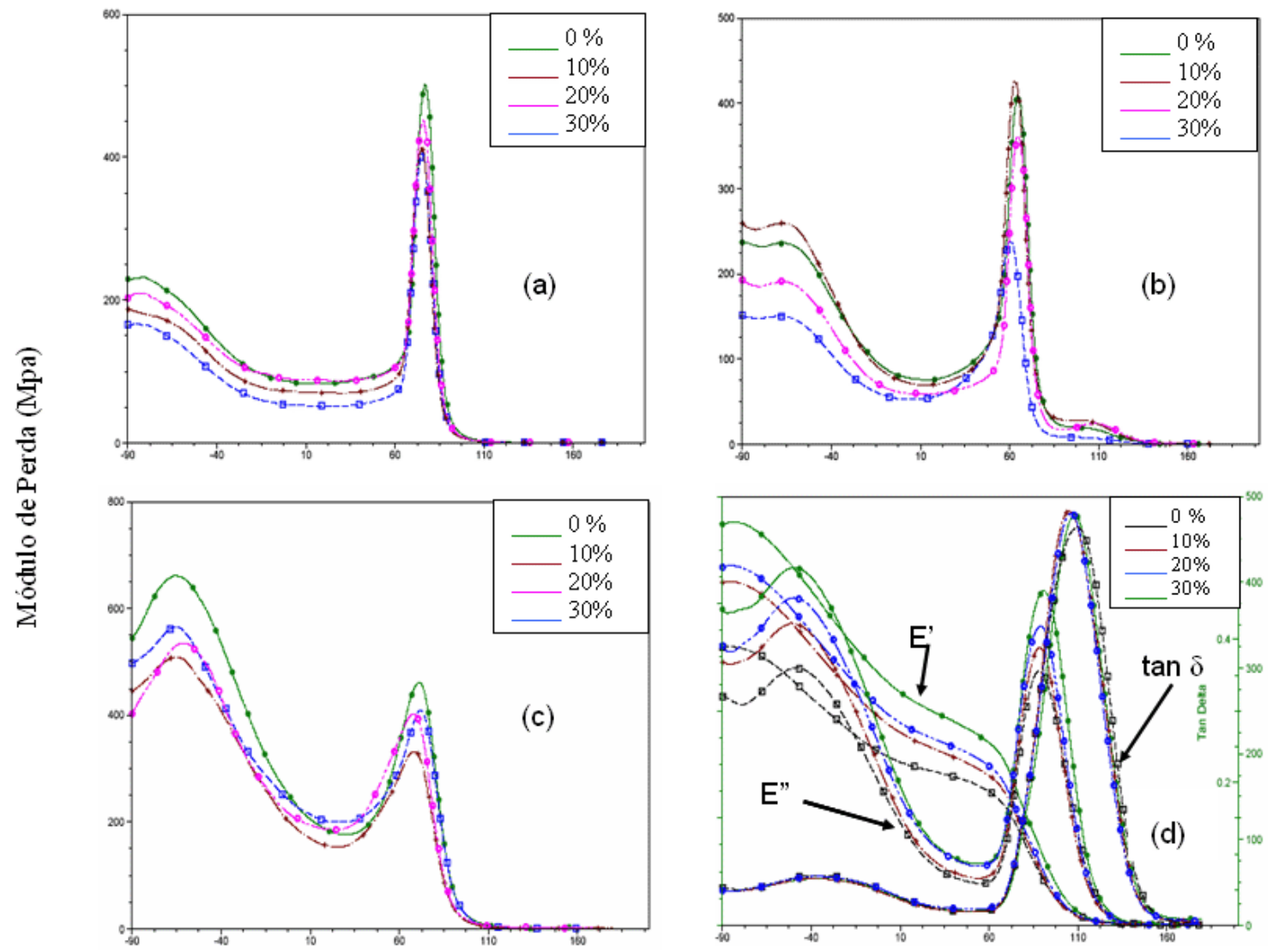

Temperatura $\left({ }^{\circ} \mathrm{C}\right)$

Figura 5: Curvas do módulo de perda vs temperatura para compósitos epoxídicos com diferentes frações em peso de partículas de diamante: (a) phr 7, (b) 13, (c) phr 17 e (d) phr 21.

A Figura 6 apresenta os conjuntos correspondentes às relações phr 7, 13, 17 e 21 de curvas de variação da tangente delta, tan $\delta$, com a temperatura para compósitos com 0, 10, 20 e 30\% em peso de diamante. O valor de $\tan \delta$, ângulo de defasagem entre as senóides dos dois módulos E” e E', corresponde à energia perdida por ciclo de deformação e está assim associado ao amortecimento ou atrito interno do material.

Observa-se na Figura 6 que, como esperado para polímeros, o valor da tan $\delta$ apresenta picos no intervalo de temperaturas pouco acima do correspondente da transição vítrea dada pelo módulo de perda na Figura 5. Isto pode ser constatado na Figura 5(d) onde estão superpostos E', E” e tan $\delta$ para o phr 21.

Assim como no caso de E”, existe praticamente uma coincidência de valores da $\tan \delta$ nos respectivos picos para cada phr. Isto indica que a introdução de partículas de diamante na matriz epoxídica causou pouca modificação no atrito interno dos compósitos. Também se verifica na Figura 6 que a menor temperatura, $73^{\circ} \mathrm{C}$, dos picos de $\tan \delta$ ocorreu para a razão estequiométrica de phr 13, enquanto que a maior temperatura dos picos, $106^{\circ} \mathrm{C}$, se deu para o phr 21, similar a E” na Figura 5.

Torna-se relevante discutir alguns resultados experimentais obtidos no presente trabalho. $\mathrm{O}$ fato dos valores de E’, Figura 3(b) e E”, Figura 5(b) para os compósitos com 10\% de diamante serem superiores até aos de $30 \%$ de diamante para o phr 13 pode ser entendido por duas hipóteses. Uma vez que o processamento de cada corpo de prova foi realizado manualmente, espera-se diferenças na distribuição das partículas de diamante $[\underline{1}, \underline{3}]$ que acarretem distorção no comportamento esperado. Outra possibilidade está relacionada com o fato dos módulos dinâmicos obtidos em ensaios de DMA serem complexos, Equação (1), e dependerem de vários fatores tais como a interação da matriz com o diamante, além da própria quantidade de partículas.

Tendo em vista os valores relativamente mais altos da intensidade dos picos de $\tan \delta$, Figura 6(b) para $10 \%$ de diamante, sugere-se que a mobilidade das cadeias moleculares na matriz esteja sendo restringida somente pelo contato físico com as partículas de diamante e não por uma efetiva interação diamante/epóxi. Caso houvesse esta interação, ocorreria uma redução do pico de $10 \%$ em relação ao de $0 \%$ de diamante [15]. 

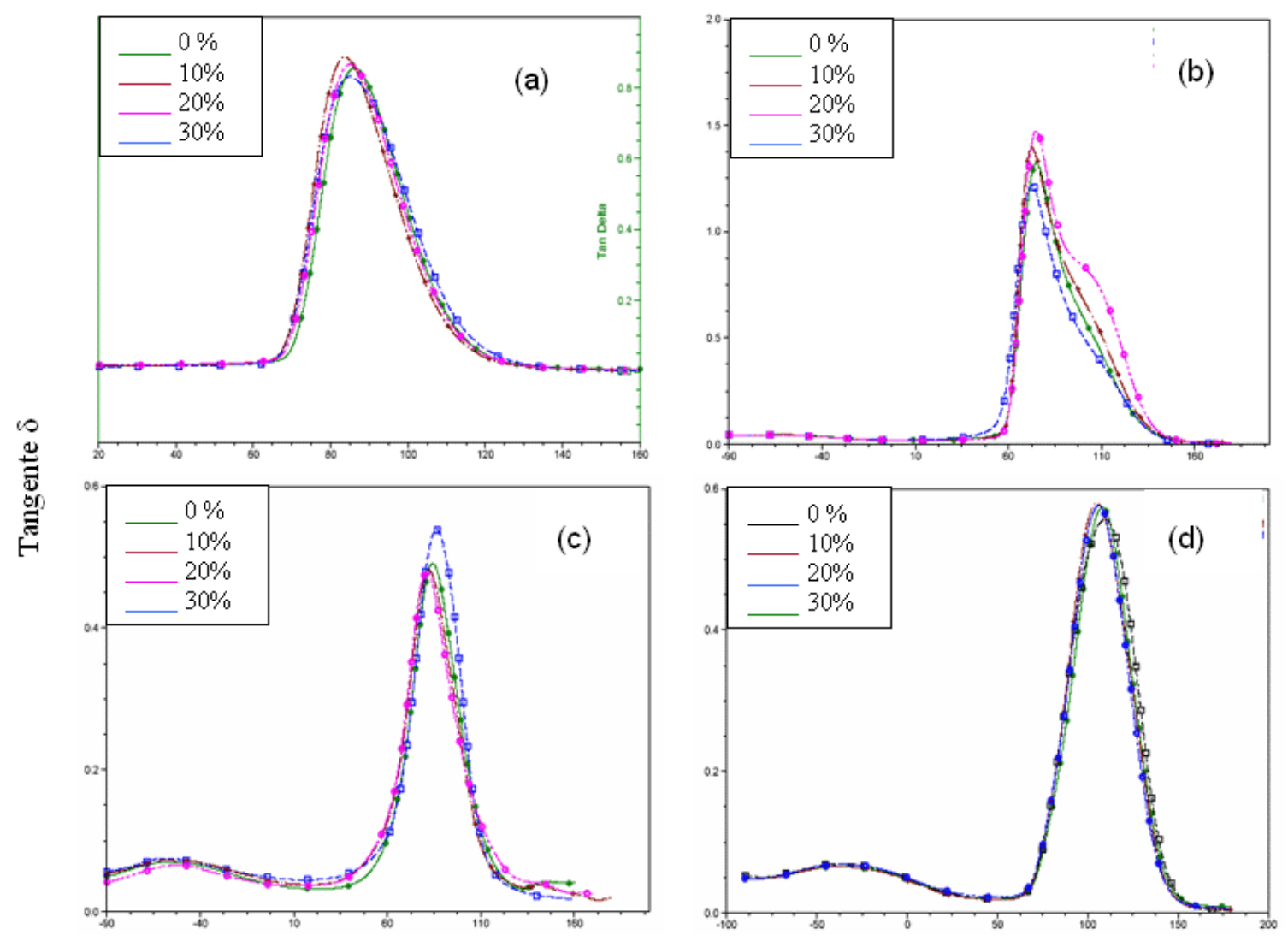

Temperatura $\left({ }^{\circ} \mathrm{C}\right)$

Figura 6: Curvas da tangente delta vs. temperatura para compósitos epoxídicos com diferentes frações em peso de partículas de diamante: (a) phr 7, (b) 13, (c) phr 17 e (d) phr 21.

No caso de phr 17, Figura 6(c), a intensidade dos picos de tan $\delta$ diminui, relativamente à pura resina epóxi, com introdução de diamante. Isto, provavelmente, está associado a alguma interação diamante/epóxi, dificultando o movimento das cadeias moleculares na matriz. Para as curvas correspondentes a phr 7, Figura 6(a), e phr 21, Figura 6(d), não parece existir indicação de uma efetiva interação. Isto justifica as maiores rigidez e tenacidade, Figuras 3 e 4, dos compósitos com phr 17.

Uma análise quantitativa da possível interação interfacial matriz/diamante pode ser conduzida através da equação proposta por Turcsányi et alli [16]:

$$
\sigma_{c}=\frac{1-\Phi}{1+A \Phi} \sigma_{m} \exp \{B \Phi\}
$$

onde $\sigma_{\mathrm{c}}$ é a resistência do compósito, $\sigma_{\mathrm{m}}$ a resistência da matriz, $\Phi$ a fração volumétrica da matriz e A e B , parâmetros do sistema.

O parâmetro A está relacionado com o formato das partículas; apresentando o valor 2,5 para partículas esféricas. Este valor será utilizado na presente análise, tendo em vista a morfologia equiaxial das partículas de diamante. Caso fosse utilizado um valor mais adequado para o formato das partículas de diamante, pouco abaixo de 2,5, não haveria diferença sensível para efeitos de comparação. O parâmetro B está relacionado com a adesão da partícula à matriz. Uma boa adesão só ocorre para $\mathrm{B} \geq 3$. Uma fraca adesão corresponde a $0<\mathrm{B}<3$. Total falta de adesão existirá para $\mathrm{B} \leq 0$ [16]. É importante mencionar que no presente trabalho a fração volumétrica, $\Phi$, foi calculada a partir da fração em peso e considerando-se a densidade do compósito. A Figura 7 apresenta a variação do valor de B com o phr da matriz para os compósitos investigados. 


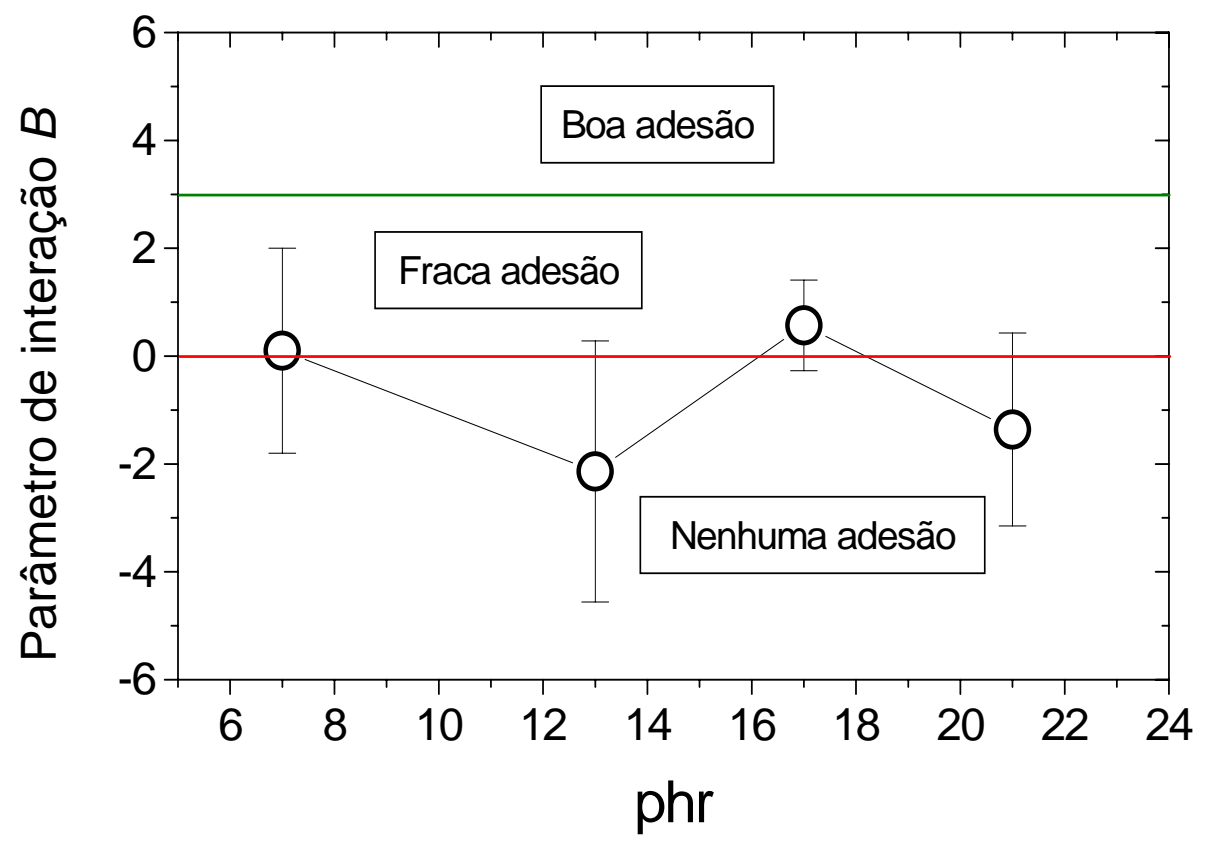

Figura 7: Variação do parâmetro de interação matriz/partícula, B, em função do phr.

A análise micrográfica por MEV tende a corroborar os resultados apresentados na Figura 7. Assim, a Figura 8 mostra partículas de diamante com evidencias de descolamento da matriz epóxi com phr 13. Esta seria, de acordo com a Figura 7, a pior condição de interação epóxi/diamante com valor médio do parâmetro de adesão B = -2,14.

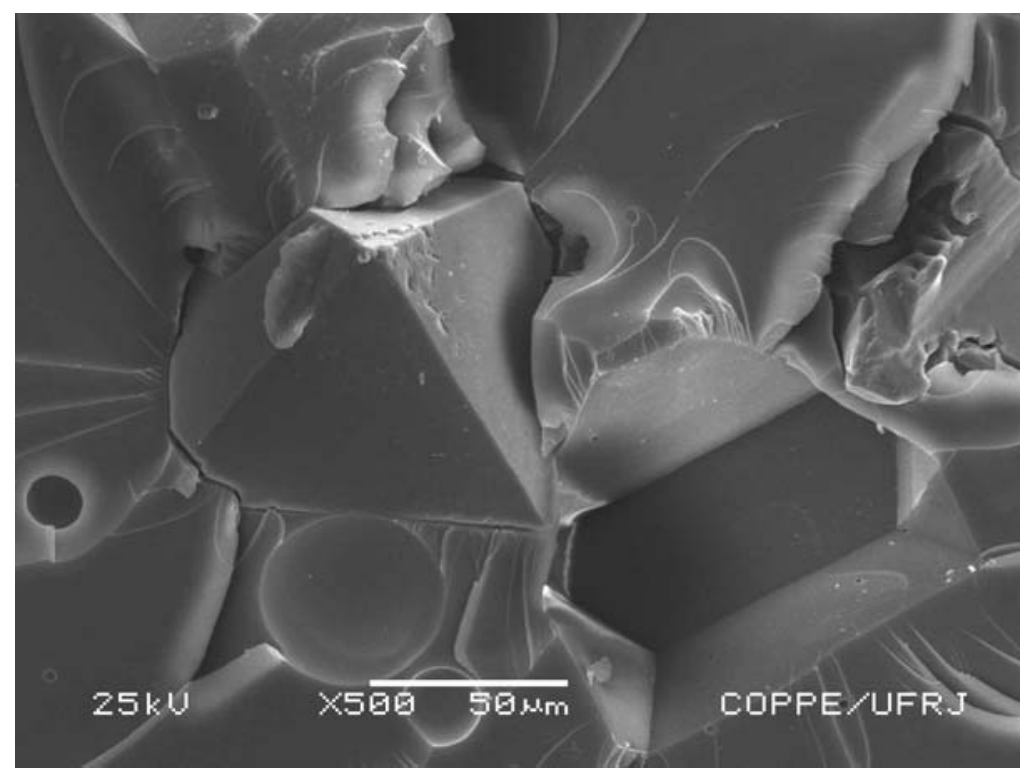

Figura 8: Partículas de diamante parcialmente aderidas em matriz com phr 13.

Por outro lado, a Figura 9 mostra que, no caso de phr 17, existe alguma interação entre a matriz e as partículas de diamante, evidenciada pela camada de resina que cobre parcialmente essa partícula. Este fato está de acordo com o valor relativamente maior de B. 


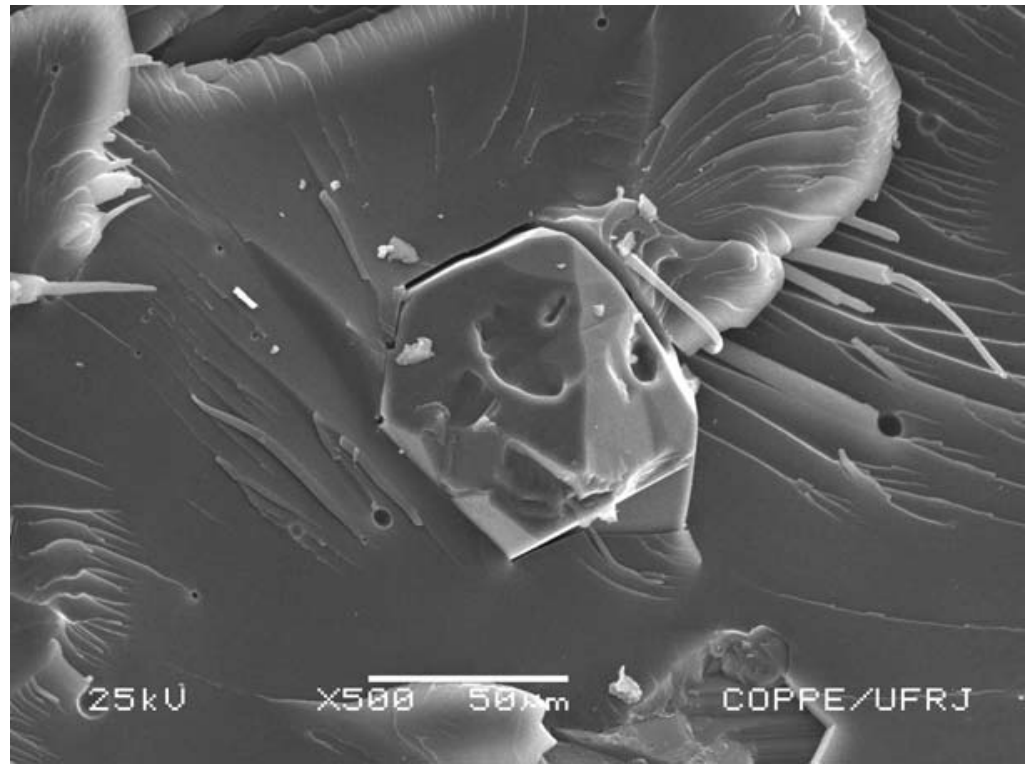

Figura 9: Partícula de diamante aderida em matriz com phr 17.

\section{CONCLUSÕES}

Resultados da resistência à tração e análise termomecânica por DMA de compósitos incorporados com partículas de diamante em matrizes epoxídicas modificadas relativamente à razão endurecedor/resina, ou seja, o valor de phr, confirmam tanto um efeito deletério destas partículas na resistência à tração quanto um aumento no módulo de armazenamento e no módulo de perda para maiores frações de diamante.

O efeito deletério pode ser interpretado através dos valores do parâmetro B de uma equação que mede a possibilidade de adesão entre partícula e matriz. Através dessa análise quantitativa associada a observações por MEV foi mostrado que o phr 13 estequiométrico apresenta a pior condição de adesão. Para os resultados de phr 17, onde se obtiveram as maiores resistências dos compósitos, constatou-se um maior valor do parâmetro de adesão. Como este parâmetro foi superior a zero, isto indicaria melhor condição de adesão, embora fraca, entre todas as resinas epoxídicas investigadas.

A temperatura de transição vítrea da matriz dos compósitos, associada aos picos obtidos tanto no módulo de perda quanto na tan $\delta$ não variou com a introdução de diamante. Uma análise da intensidade dos picos da tan $\delta$ sugere que nos compósitos com razão estequiométrica, phr 13, não exista interação interfacial diamante/matriz. Já para o phr 17 existem evidências de interação, o que poderia justificar a maior rigidez e tenacidade destes compósitos.

\section{AGRADECIMENTOS}

Os autores agradecem o apoio a esta pesquisa na forma de auxílios e bolsas concedidas pelo CNPq, CAPES e FAPERJ. É também motivo de agradecimento a colaboração prestada pelo Prof. José Roberto Moraes d'Almeida da PUC-Rio, bem como a permissão do PEMM/COPPE/UFRJ de utilizar o MEV e a colaboração de Márcia Soares Sader e Felipe Sabatino Gonçalves Vieira.

\section{BIBLIOGRAFIA}

[1] MONTEIRO, S.N., MENEZES, G.W., RODRIGUEZ, R.J.S., SKURY, A.L.D., BOBROVNITCHII, G.S., "Processing and Mechanical Behavior of Diamond Reinforced DGEBA/TETA Epoxy Matrix Composites”, In: PPS-2004, Americas Regional Meeting, pp. 108-109, Florianópolis, Brasil, 2004.

[2] MENEZES, G.W., MONTEIRO, S.N., D’ALMEIDA, J.R.M., “Comportamento Mecânico de Compósitos Diamantados com Matriz Epóxi”, In: 60 Congresso Anual da ABM, pp. 1-9, Belo Horizonte, Brasil, 2005. 
[3] MONTEIRO, S.N., MENEZES, G.W., RODRIGUEZ, R.J.S., SKURY, A.L.D., BOBROVNITCHII, G.S., "Effect of Diamond Particles in the Fracture of an Epoxy Composite", In: Jornadas SAM/CONAMET 2005, pp. 1-7, Mar de Plata, 2005.

[4] MONTEIRO, S.N., MENEZES, G.W., RODRIGUEZ, R.J.S., BOBROVNITCHII, G.S., SKURY, A.L.D., “Technical Characteristics of Diamond Reinforced Epoxy Composites”, Revista Matéria, v. 10, n. 4, pp. 5564-570, 2005.

[5] DE NOGRARO, F.F., GUERRERO, P., COCUERA, M.A., MONDRAGÓN, I., "Effects of Chemical Structure of Hardener on Curing Evolution and on the Dynamic Mechanical Behavior of Epoxy Resins”, Journal of Applied Polymer Science, v. 56, pp. 177-192, 1995.

[6] MEYER, F.; SANZ, G., ECEIZA, A., MONDRAGÓN, I., MIJOVIC, J., "The Effect of Stoichiometry and Thermal History during Cure on Structure and Properties of Epoxy Networks”, Polymer, v. 36, pp. 1407-1414, 1995.

[7] D'ALMEIDA, J.R.M., MONTEIRO, S.N., “The Influence of the Amount of Hardener on the Tensile Mechanical Behavior of an Epoxy System”, Polymers for Advanced Technologies, v. 9, pp. 216221, 1998.

[8] SHIMBO, M, NISHITANI, N., TAKAHAMA, T., "Mechanical properties of Acid-Cured Epoxide Resins with Different Network Structures”, Journal of Applied Polymer Science, v. 29, pp. 1709-1721, 1984.

[9] D’ALMEIDA, J.R.M., MONTEIRO, S.N., “The influence of the Hardener/Resin Ratio on the Impact Behavior of an Epoxy System”, Journal of Polymer Engineering, v. 18, pp. 235-248, 1998.

[10] D’ALMEIDA, J.R.M., MONTEIRO, S.N., “Analysis of the Fracture Morphology of an Epoxy System as a Function of the Resin/Hardener Ratio”, Journal of Materials Science Letters, v. 15, pp. 955958, 1996.

[11] MONTEIRO, S.N., MENEZES, G.W., D’ALMEIDA, J.R.M., RODRIGUEZ, R.J.S., "Processing and Thermal Analysis of DGEBA/TETA Epoxy Systems with Different Hardener/Resin Ratios”, In: PPS-2004, Americas Regional Meeting, pp. 262-263, Florianópolis, Brasil, 2004.

[12] SKURY, A.L., BOBROVNITCHII, G.S., MONTEIRO, S.N., "Experimental Design Methodology Applied to Study a Diamond Purification Process”, Diamond and Related Materials, v. 13, pp. 1638-1644, 2004

[13] NOWICK, A.S., BERRY, B.S., Anelastic Relaxation in Crystalline Solids, Nova York, EUA, Academic Press, 1972.

[14] FERRY, J.D., Viscoelastic Properties of Polymers, Nova York, EUA, John Wiley \& Sons, 1980.

[15] NAIR, K.C.M., THOMAS, S., GROENINCKX, G., “Thermal and Dynamic Mechanical Analysis of Polystyrene Composites Reinforced with Short Sisal Fibres”, Composites Science and Technology, v. 61, pp. 2519-2529, 2001.

[16] TURCSÁNYI, B., PUKÁNSKY, B., TÜDOS, F., “Composition Dependence of Tensile Yield Stress in Filled Polymers”, Journal of Materials Science Letters, v. 7, pp. 160-162, 1988. 